\title{
HIV Post-Exposure Prophylaxis Use and Associated Factors among Health Professionals of Governmental Health Institutions in Mekelle Town, Tigray Ethiopia, Cross-Sectional Study
}

Tsegabrhan Gebreslase ${ }^{1 *}$ and Gerezgiher Buruh ${ }^{2}$

${ }^{1}$ Humera Hospital, ART center, Tigray, Ethiopia

${ }^{2}$ Department of Nursing, Mekelle University, Tigray, Ethiopia

\begin{abstract}
Background: Infection with Human Immunodeficiency Virus is a serious public health problem, costing the lives of many people including health workers. Health care workers practicing in developing countries like Ethiopia are more exposed to HIV following occupational exposure and less likely to use post exposure prophylaxis. Ethiopia has developed guidelines on the prevention of infection in health institutions in July 2004 and also employed the use of post exposure prophylaxis since the implementation of free antiretroviral in January 2005.
\end{abstract}

Objective: the main aim of this study was to assess prevalence of PEP service use among health professionals in Mekelle town.

Methodology: A health institution based cross sectional study design involving 190 health professionals was employed using a structured self administered questionnaire. Sampling technique was based on the population proportion to size, then to select a study unit systematic random sampling was used. SPSS version 16.00 was used for data entry and analysis. Proportions and percentages were used for descriptions of data.

Result: the study revealed that occupational exposure to blood, non bloody body fluids, needle stick injuries and mucocutaneous were $82.5 \%, 74.9 \%, 49.1 \%$ and $42.7 \%$ respectively. Among the exposed health professionals $19.6 \%$ use PEP. The main reasons for not using PEP was source patient HIV test result negative $(65.5 \%)$, followed by negligence (25\%). For those who started PEP all of them get HIV testing before commencing PEP and $80 \%$ of them completed in 4 weeks, $20 \%$ discontinue the PEP due to adverse effects of the drugs. Training of health professionals on PEP had statistically significant association with PEP utilization (AOR=2. 864, 95\% $\mathrm{Cl}=1.152-7.122)$

Conclusion: the finding of this result indicated that occupational exposures were common among health professionals. The use of PEP among exposed health professionals was low. Providing training for all health professionals on infection prevention, including PEP is recommended to lower the occupational exposure and to enhance the use of PEP.

Keywords: Occupational exposure; Health professionals; Post exposure prophylaxis; Mekelle

\section{Introduction}

HIV infection is a pandemic, serious public health problem, as of December 2007, 33 million people were estimated to be living with HIV/ AIDS. Of the 33 million, 22.5 million were living in sub-Saharan Africa alone, where the adult prevalence rate is 5.0 percent $[1,2]$. It is estimated that $4.4 \%$ (range $0.8 \%$ to $18.5 \%$ ) of all HIV infections amongst HCWs are due to occupational injuries. It is further estimated that at least half of these cases occur in sub-Saharan Africa [3,4].

The adoption of standard universal precaution and use of personal protective equipments (PPEs) led to a significant reduction in occupational exposures over the last two decades. Despite these precautions, occupational exposures are still occurring and the use of PEP is paramount [5]. Prophylaxis were primarily used after occupational exposures of health care workers to HIV-infected blood and body fluids, usually through needle stick injuries or contact with splashes blood or body fluids $[3,6,7]$.

Occupational exposure to HIV is probably the most serious causes of highest level anxiety amongst health professionals in many countries including in Ethiopia. Ethiopia is one of the hardest hit countries by HIV/ AIDS epidemics with the national HIV prevalence $2.4 \%$ of adults $[1,8]$.
Occupational infection from body fluid borne pathogens is the most serious health risk faced by healthcare workers. Of 35 million healthcare workers worldwide, the World Health Organization (WHO) estimated that approximately 3 million experiences percutaneous injuries each year. Most of these exposures occur in developing countries, where the prevalence of body fluid borne pathogens in the general population is high and access to protective equipment is limited [9].

Exposures that place a health care worker at potential risk of HIV infection are percutaneous injuries (a needle stick or cut with a sharp object) or contact of mucous membrane or non intact skin (exposed skin that is chapped, abraded, or afflicted with dermatitis) with blood, tissue, or other potentially infectious body fluids. HIV transmission following skin puncture from a needle or a sharp object that was contaminated

*Corresponding author: Gerezgiher Buruh Abera, Department of Nursing, College of Health Sciences, Mekelle University, P. Box 1871, Tigray region, North Ethiopia, Tel: 0344416681; E-mail: gbamsc2002@gmail.com, loveingo@yahoo.com

Received April 21, 2014; Accepted May 20, 2014; Published June 04, 2014

Citation: Gebreslase T, Buruh G (2014) HIV Post-Exposure Prophylaxis Use and Associated Factors among Health Professionals of Governmental Health Institutions in Mekelle Town, Tigray Ethiopia, Cross-Sectional Study. J AIDS Clin Res 5: 313. doi:10.4172/2155-6113.1000313

Copyright: (c) 2014 Gebreslase T, et al. This is an open-access article distributed under the terms of the Creative Commons Attribution License, which permits unrestricted use, distribution, and reproduction in any medium, provided the original author and source are credited. 
with blood from a person with documented HIV infection is $~ 0.3 \%$, and after a mucous membrane exposure is $0.09 \%[9,10]$.

After exposure, HIV replicates within dendritic cells of the skin and mucous before spreading through the lymphatic vessels and develops into systemic infection. This delay in the systemic spread gives an opportunity for PEP using designed to block replication of HIV [11]. Even though the maximum delay for initiation of treatment to prevent infection is not known in human. The CDC recommendations are to offer prophylaxis up to 24 to 36 hours after exposure (No consideration of PEP beyond 72 hours) $[2,11,12]$.

The drugs used for PEP can be 2 (low risk exposure) or 3 (for high risk exposure) anti retroviral therapy (ART) for 28 days. Conditions like blood come into contact with cuts on the skin, exposure to a large volume of blood or potentially infectious fluids or blood contamination from terminally ill patients or early seroconversion phase of HIV, injury with a hollow needle and deep and extensive injury are considered as high risk exposure [4-8].

The low risk exposure is considered a small volume of blood, blood contamination from asymptomatic HIV positive patients, injury with solid needle, superficial injury, glove use during exposure and mucocutaneous exposures. The health professionals at high risk of occupational exposure include physicians, health officers, nurses, midwives and laboratory technicians. When used properly PEP reduces the rate of HIV infection from exposure by $79-81 \%[1,2,11,12]$.

Health care workers practicing in poor countries like Ethiopia are more exposed to HIV occupational exposure and less likely to use PEP than those working in developed countries [4]. A research done on the assessment of HIV post-exposure prophylaxis use among health workers of governmental health institutions in Jimma zone, Oromiya region, southwest Ethiopia by Bosena Tebeje, in 2010 showed that $142(81.6 \%)$ of those exposed reported that they did not use PEP [1]. This research will, therefore, try to explore the existing magnitude of occupational exposure to HIV and use of PEP among those exposed to health professionals in Mekelle town.

HIV post exposure prophylaxes are a form of secondary HIV prevention that may reduce the incidence of HIV infections. Occupational HIV PEP is an accepted form of therapy for health care workers exposed to HIV through their jobs. Since its inception, the medical profession has been vulnerable to occupational exposure to infectious diseases. With the emergence of HIV infection and its relentless global spread, health care workers are increasingly being exposed to patients who are HIV positive or have frank AIDS. Despite the use of universal precautions, accidental occupational exposure to blood or potentially infectious materials does occur [13].

The risk for occupational exposure to HIV has been well characterized in the developed world, but limited information is available about this transmission risk in resource-constrained settings facing the largest burden of HIV infection. In addition, the feasibility and utilization of post-exposure prophylaxis programs in these settings are unclear [5].

Ethiopia as one of the resource constrained countries, the magnitude of occupational exposure and utilization of PEP among health professional is not known. Specifically, in Mekelle published studies showed that the clear picture of occupational exposure and HIV PEP use in the workplace does not exist. This study is therefore undertaking to assess the level of occupational exposure and knowledge, attitude, practice; and factors associated with HIV PEP use among health professionals working in governmental health institutions in Mekelle town. Finally to come up with recommendations to enable responsible bodies to design, integrate and to take appropriate measure. It will also help as an input for the subsequent large scale researches.

\section{Methods}

\section{Study area and period}

The study was undertaken in Mekelle town from July 23 to August 03 2012. Mekelle is the capital city of Tigray and found around 780 $\mathrm{km}$ away the capital city Addis Ababa. The current total population of Mekelle is estimated to be 264,907 (2011). According to the existing health service delivery system, the town has 4 governmental, 4 private hospitals, and 9 health centers, 22 private clinics, 6 rural drug venders, 35 drug shops 4 pharmacies and 9 whole sellers. There are about 806 health professionals from these 83 physicians, 24 health officers, 482 nurses, 60 laboratory technicians and 38 midwives working in these health centers and hospitals.

An institution based cross sectional study design was employed between July 23 to August 032012.

The study population was health professionals who were currently working in two hospitals and three health centers, which provide ART in the town and directly involved in the care of patients (Physicians, health officers, nurses, midwives and laboratory technicians) in hospitals and health centers of the study area.

The inclusion criteria were all health professionals working in the study area who have a potential to be exposed to HIV high risk conditions in their day to day professional activities; and the exclusion criteria are health professionals in whom their day to day activity doesn't make them to be at high risk of HIV due to occupational exposure.

The sample size was determined using single proportion formula; with a $\mathrm{P}$ value taken from previous studies in the local context in Bahirdar town, 2010; which was 19\% [11]. Since the source population was less than 10,000 a finite population correction formula was used; therefore, the final sample size was 190 . To identify the study subject a systematic random sampling was applied to each health facility. From the list of all the source populations, Kth value was calculated; then every Kth person was included in the study. To select the first study subject lottery method was used.

Data collection was conducted using a structured self administered questionnaire. Before the actual data collection began, pretest was done in Quiha hospital and Quiha health center in 5\% of the sample. After data collection was completed, each questionnaire was checked for completeness, missing values and unrelated responses. The coded data were entered into the computer using Epi-Info and cleaning were performed, then the cleaned data was exported to SPSS version 16.0 for analyses.

Ethical clearance was obtained from Mekelle University, college of health science Ethical Review Committee and Tigray Health Bureau. The letter was written to medical director of Mekelle hospital and head of the health centers to obtain their consent. Necessary explanation about the purpose of the study and its procedures was done to the respondent and their consent was also obtained from each respondent. To ensure confidentiality, anonymity was used and they were informed to withdraw at any time if inconvenient.

\section{Results}

\section{Socio-demographic characteristics of the study population}

The study was conducted on 190 health care professionals with a 


\begin{tabular}{|c|c|c|}
\hline Characteristics & & Frequency \\
\hline \multirow{4}{*}{ Age } & $20-29$ & $91(53.2)$ \\
\hline & $30-39$ & $45(26.3 \%)$ \\
\hline & $40-49$ & $25(14.6 \%)$ \\
\hline & $50-59$ & $10(5.8 \%)$ \\
\hline \multirow{2}{*}{ Sex } & Male & $62(36.3 \%)$ \\
\hline & Female & $109(63.7 \%)$ \\
\hline \multirow{5}{*}{ Marital status } & Single & $81(47.4 \%)$ \\
\hline & Married & $74(43.3 \%)$ \\
\hline & Divorced & $11(6.4 \%)$ \\
\hline & Widowed & $3(1.8 \%)$ \\
\hline & Separated & $2(1.2 \%)$ \\
\hline \multirow{4}{*}{ Religion } & Orthodox & $162(94.7 \%)$ \\
\hline & Muslim & $5(2.9 \%)$ \\
\hline & Protestant & $2(1.2 \%)$ \\
\hline & Catholic & $2(1.2 \%)$ \\
\hline \multirow{5}{*}{ Profession } & Nurses & $106(62 \%)$ \\
\hline & Midwife & $24(14 \%)$ \\
\hline & Physician & $16(9.4 \%)$ \\
\hline & Laboratory & $14(8.2 \%)$ \\
\hline & Health officer & $11(6.4 \%)$ \\
\hline \multirow{6}{*}{ Department } & Medical ward & $34(19.9 \%)$ \\
\hline & Outpatient department & $50(29.9 \%)$ \\
\hline & Gynecology \& obstetrics & $35(20.5 \%)$ \\
\hline & Pediatrics & $20(11.7 \%)$ \\
\hline & Surgical ward & $17(9.9 \%)$ \\
\hline & Laboratory & $15(8.8 \%)$ \\
\hline \multirow{2}{*}{ Service year } & $<10$ years & $119(69.6 \%)$ \\
\hline & $>10$ years & $52(30.4 \%)$ \\
\hline \multirow{2}{*}{ Night shift } & Yes & $136(79.5 \%)$ \\
\hline & No & $35(20.5 \%)$ \\
\hline \multirow{2}{*}{ Patient cared daily } & $<35$ & $126(73.7 \%)$ \\
\hline & $>35$ & $45(26.3 \%)$ \\
\hline
\end{tabular}

Table 1: Socio demographic characteristics of the respondents.

non response rate of $10 \%$ (19 questionnaires, 15 not returned and 4 incorrectly filled). A majority, 109 (63.7\%), of the respondents were females. The age of the respondents ranged from 20 to 58 years with the median age of 28 years.

Of the total respondents, $106(62 \%)$ were nurses, $24(14 \%)$ midwives, $16(9.4 \%)$ physicians, $14(8.2 \%)$ laboratory and $11(6.4 \%)$ were health officers. Almost all of the participants, 162 (94.7\%), were Orthodox Christians and 81 (47.4\%) were single in marital status.

About $79.5 \%$ of the respondents were working in night shifts and $126(73.3 \%)$ of the respondents cared less than 35 patients per day the rest $45(26.3 \%)$ cared greater than 35 patients per day (Table 1).

\section{Perception of professional HIV infection risk}

Out of the total respondent $145(84.8 \%)$ had perceived risk of acquiring HIV infection. 83 (48.5\%) respondents leveled themselves as having high risk and 63 (36.8\%) leveled themselves as having low risk for HIV while 25 (14.6\%) don't know whether they are or not at risk of HIV infection.

\section{Prevalence of occupational exposures among health professionals}

Blood was the most common fluid in which health professionals were exposed during their day to day activities. The exposure to blood, non blood body fluid, needle stick injury and mucocutaneous were $82.5 \%, 74.9 \%, 49.1 \%$ and $42.7 \%$ respectively.

\section{Health professional's exposure to HIV risk conditions}

Among the 84 needle stick injuries, $69(82.1 \%)$ had $1-3$ times in their lifetime, 13 (15.47\%) had 4-6 times in their lifetime and $2(2.3 \%)$ had 7-10 times stick injuries in their lifetime. Based on the last year history of exposure $48(28.07 \%)$ of the respondents had at least one needle stick injury.

The most common reason for sustaining the recent injury was due to patient sudden movement $21(43.7 \%)$, during recapping $13(27 \%)$, during sharp collection eight $(16.6 \%)$ and due to other causes seven (14.6\%) (Table 2).

\section{Immediate Measures taken after needle stick injury and mucosemembrane exposure}

Different measures were taken by health professional immediately following exposure. Washing with soap and water was the most common measure taken $47(55.9 \%)$ followed by squeezing for more bleeding 10(11.9\%).

Respondents answered different measure after body fluid exposure to their eye, mouth and/nose. The majority $61(83.56 \%)$ considered immediate washing with water and soap $61(83.56 \%)$, visiting VCT immediately after exposure five (6.8\%) (Table 3).

\section{Knowledge of health professionals about HIV PEP}

Almost all of the respondents 167 (97.7\%) knew the presence of PEP drugs that are given following accidental occupational exposure to HIV, $160(93.3 \%)$ of the respondents answered that HIV testing is important before commencing PEP drugs against HIV. 53 (31\%) of the respondents replied that either two or three PEP drugs can be used based on level of exposure. 93 (54.4\%) of the respondents answered that the maximum time limit to initiate HIV PEP is 72 hours and 140 $(81.9 \%)$ of the respondents answered that 28 days is the time duration for PEP.

In general $98(57.3 \%)$ of the respondents had good knowledge $71(41.5 \%)$ had fair knowledge and 2(1.2\%) had poor knowledge about PEP (Table 4).

\section{Training of professionals on pep and availability of pep at} work places

Among the respondents 53 (31\%) have trained on PEP. 118 (69\%) don't take any training on PEP, 73 (42.7\%) of the respondents replied that they have guidelines and protocols on how to use PEP after exposure, 98 (57.3\%) they don't have guidelines and protocols of PEP.

$117(68.4 \%)$ of the respondents respond that PEP is available at working hour, 23(13.5\%) answered that PEP is not available at workings hours, 31(18.1\%) don't know the availability of PEP.

$72(42.1 \%)$ of the respondents respond that PEP was available on weekend days and 58 (33.9\%) respond that PEP not available at weekend days. The remaining 41 (24\%) don't know the availability of PEP at weekend days.

\section{PEP use among health professionals}

Regarding exposure to the risk of acquiring HIV/AIDS 158 (92\%) of the 171 health professionals exposed to HIV risk conditions. However, only 31 (19.6\%) of the 158 exposed use PEP. The main reason reported for not using PEP were source patient HIV test result negative $76(65.5 \%)$, negligence $29(25 \%)$ and unaware of the existence of PEP six (5.1\%) (Table 5). 


\begin{tabular}{|c|c|c|c|c|c|c|c|c|}
\hline \multirow{3}{*}{ profession } & \multicolumn{8}{|c|}{ HIV risk conditions } \\
\hline & \multicolumn{2}{|c|}{ Body fluid } & \multicolumn{2}{|c|}{ blood } & \multicolumn{2}{|c|}{ Needle stick } & \multicolumn{2}{|c|}{ Mucous membrane } \\
\hline & Yes & No & Yes & No & Yes & No & Yes & No \\
\hline Physician(16) & $13(81.3 \%)$ & $3(18.7 \%)$ & $13(81.3 \%)$ & $3(18.7 \%)$ & $11(68.7 \%)$ & $5(31.3 \%)$ & $8(50 \%)$ & $8(50 \%)$ \\
\hline Health officer(11) & $9(81.8 \%)$ & $2(18.2 \%)$ & $8(72.7 \%)$ & $3(27.3 \%)$ & $5(45.4 \%)$ & $6(54.6 \%)$ & $7(63.3 \%)$ & $4(36.7 \%)$ \\
\hline Nurse (106) & $77(72.6 \%)$ & $29(27.4 \%)$ & $86(81.1 \%)$ & $20(18.9 \%)$ & $54(50.9 \%)$ & $52(49.1 \%)$ & $39(36.8 \%)$ & $67(63.2 \%)$ \\
\hline Midwife(24) & $23(95.8 \%)$ & $1(4.2 \%)$ & $21(87.5 \%)$ & $3(12.5 \%)$ & $8(33.3 \%)$ & $16(66.7 \%)$ & $17(70.8 \%)$ & $7(29.2 \%)$ \\
\hline Laboratory(14) & $6(42.8 \%)$ & $8(57.3 \%)$ & $13(92.8 \%)$ & $1(7.2 \%)$ & $6(42.8 \%)$ & $8(57.2 \%)$ & $2(14.3 \%)$ & $12(85.7 \%)$ \\
\hline
\end{tabular}

Exposure to body fluid at $\mathrm{p}$ value $=.007$

Exposure to blood $\mathrm{p}=0.675$

Exposure to needle $p=0.260$

Exposure to mucosemembrane $\mathrm{p}=.003$

Table 2: Health professional's exposure to HIV risk conditions in Mekelle town Occupational exposure by category of professions.

\begin{tabular}{|c|c|c|c|c|}
\hline \multirow{2}{*}{ measurements } & \multicolumn{2}{|c|}{ needle stick injury } & \multicolumn{2}{|c|}{ mucosememrane } \\
\cline { 2 - 5 } & Frequency & Percent & 61 & Percent \\
\hline wash with soap and water & 47 & 55.9 & 3 & 0 \\
\hline wash with alcohol and iodine & 7 & 8.3 & 5 & 4.1 \\
\hline squeeze for more bleeding & 10 & 11.9 & 2 & 00 \\
\hline visiting VCT & 7 & 8.3 & 0 & 2.7 \\
\hline seek PEP & 7 & 8.3 & 1 & 0 \\
\hline report to head person & 1 & 1.2 & 1 & 2.7 \\
\hline doing nothing & 4 & 4.76 & 73 & 1.4 \\
\hline Other & 1 & 1.2 & 73 & 100 \\
\hline Total & 84 & 100.0 & & \\
\hline
\end{tabular}

Table 3: Measures taken immediately after exposure.

\begin{tabular}{|c|c|c|c|}
\hline Question & Response & Frequency & Percent \\
\hline \multirow{2}{*}{ Knowing the presence of PEP } & Yes & 167 & 97.7 \\
\hline & No & 4 & 2.3 \\
\hline \multirow{2}{*}{ HIV testing before starting PEP } & Yes & 160 & 93.6 \\
\hline & No & 11 & 6.4 \\
\hline \multirow{3}{*}{ Naming of PEP } & Correctly mentioned & 53 & 31 \\
\hline & Wrongly mentioned & 49 & 28.7 \\
\hline & Mentioned nothing & 69 & 40.4 \\
\hline \multirow{3}{*}{ Maximum time limit to initiate PEP } & Correct answer & 93 & 54.4 \\
\hline & Wrong answer & 67 & 39.2 \\
\hline & Don't know & 11 & 6.4 \\
\hline \multirow{3}{*}{ Duration of PEP } & Correct answer & 140 & 81.9 \\
\hline & Wrong answer & 14 & 8.2 \\
\hline & Don’t know & 17 & 9.9 \\
\hline
\end{tabular}

Table 4: knowledge of health professionals to PEP.

\begin{tabular}{|c|c|c|}
\hline Reasons for not using PEP & Frequency & Percent \\
\hline Source patient HIV test results become negative & 76 & 65.5 \\
\hline Negligence & 29 & 25 \\
\hline Unaware of presence of PEP & 6 & 5.12 \\
\hline Lack of understanding the value of PEP & 3 & 2.58 \\
\hline Fear of stigma and discrimination & 1 & 0.8 \\
\hline Uncertaininty about confidentiality & 1 & 0.8 \\
\hline Total & 116 & 100 \\
\hline
\end{tabular}

Table 5: Reasons for why the health professional didn't use PEP.

For those who received PEP 31 (100\%) they receive HIV counseling and testing before they start PEP, 12 (38.7\%) of these started PEP they start the first PEP with less than 6hours, 6 (19.4\%) started with 7-12 hours, 12 (38.7\%) started with in $13-24$ hours, one (3.2\%) respond that he or she started the first PEP 25-48 hours. The mean time to initiate PEP among the respondents was 13.35 hours 25 (80.6\%) of those who started PEP completed their treatment, 6 (19.4\%) discontinued their PEP. 5 (83.3\%) of those who discontinued are due to adverse effect of the drugs and $1(16.7 \%)$ was due to source patient turned to be HIV negative.

29 (93.5\%) of those received PEP get HIV testing at 3 and 6 months after completion of the PEP on these 28 become negative and one become HIV positive.

\section{Factors associated with utilization of PEP}

Factors associated with Post exposure prophylaxis use were assessed 


\begin{tabular}{|c|c|c|c|c|}
\hline \multicolumn{2}{|l|}{ Variable } & \multirow{2}{*}{\begin{tabular}{|c|} 
P-value \\
.919
\end{tabular}} & \multirow{2}{*}{$\begin{array}{c}\text { COR(95\% Cl) } \\
.919(.179-4.722)\end{array}$} & \multirow{2}{*}{$\mathbf{X}^{2}$} \\
\hline \multirow{4}{*}{ Age } & $20-29$ & & & \\
\hline & $30-39$ & .591 & $.615(.105-3.620)$ & \multirow{3}{*}{1.299} \\
\hline & $40-49$ & .799 & $1.263(209-7.649)$ & \\
\hline & $50-59$ & & 1 & \\
\hline \multirow{2}{*}{ Sex } & Male & .921 & $.960(.426-2.162)$ & \multirow{2}{*}{.010} \\
\hline & Female & & 1 & \\
\hline \multirow{5}{*}{ Marital status } & Single & .999 & 1 & \multirow{5}{*}{2.271} \\
\hline & Married & .999 & $3.44(.4432-2.3446)$ & \\
\hline & Divorced & .999 & $9.23(.7012-10.664)$ & \\
\hline & Separated & 1 & - & \\
\hline & Widowed & & - & \\
\hline \multirow{5}{*}{ Profession } & Physician & .076 & 7.8(.804-75.640) & \multirow{5}{*}{5.121} \\
\hline & Health officer & 414 & $2.889(.226-36.868)$ & \\
\hline & Nurse & .396 & $2.483(.304-20.259)$ & \\
\hline & Midwife & .286 & $3.421(.357-32.783)$ & \\
\hline & Laboratory & & 1 & \\
\hline \multirow{3}{*}{ Risk of HIV infection } & Yes & .521 & $2(.24-16.634)$ & \multirow{3}{*}{2.088} \\
\hline & No & 639 & $.5(.028-9.076)$ & \\
\hline & I don't know & & 1 & \\
\hline \multirow{2}{*}{ Training on PEP } & Yes & .008 & $2.969(1.336-6.597)$ & \multirow{2}{*}{7.14} \\
\hline & No & & 1 & \\
\hline \multirow{2}{*}{ Having guide line on PEP } & Yes & 621 & $.818(.369-1.813)$ & \multirow{2}{*}{.245} \\
\hline & No & & 1 & \\
\hline \multirow{3}{*}{ Score of knowledge } & Good & .3 & $.225(.013-3.769)$ & \multirow{3}{*}{1.211} \\
\hline & Fair & .272 & $.203(.012-3.483)$ & \\
\hline & Poor & & 1 & \\
\hline \multirow{2}{*}{ Service year cat } & $<10 y e a r$ & .854 & $1.084(.461-2.547)$ & \multirow{2}{*}{.034} \\
\hline & >10year & & 1 & \\
\hline \multirow{2}{*}{ Patient cared daily } & $<35$ & .602 & $1.277(.509-3.207)$ & .272 \\
\hline & $>35$ & & 1 & \\
\hline
\end{tabular}

NB * shows significant association

- Implies no event

Table 6: Bivariate analysis of socio demographic \& behavioral factors associated PEP utilization in Mekelle town

based on the developed conceptual framework. Cross tabulation was first used to assess the association between dependent and independent variables. Variables which reach a $\mathrm{P}$ value of less than 0.05 were considered as having associated with PEP utilization. Accordingly, no independent variable had statistically significant association with PEP utilization ( $p>0.05$ ) except training on PEP. Then binary logistic regression was used to show the associations as shown in the following Table 6.

The Bivariate analysis was performed using chi square (x2) test. Accordingly, no independent variable was statistically significant $(\mathrm{P}<0.05)$ except training on PEP. The odds of PEP utilization were 3times higher among those who trained on PEP when compared with those who were not trained on PEP $(\mathrm{COR}=2.969,95 \% \mathrm{CI}=1.336$ 6.597).

Multivariate logistic regression analysis was also done to see the association among variables. According to the multivariate logistic regression analysis result, ever trained on PEP was found to have significant association with PEP utilization $(\mathrm{AOR}=2.864,95 \%$ CI 1.152 -7.122). On other variables, the analysis did not show an association (Table 7).

\section{Discussion}

This study assessed prevalence of occupational exposure to HIV, post exposure prophylaxis use among health professionals and factors associated with utilization of PEP in Mekelle town.

This study detected high levels of occupational exposures. Ever needle stick and sharp injuries (49.1\%) and $28.07 \%$ experienced injury in the last year. This is greater than with previous similar studies done in Ethiopia in Addis Ababa on Occupational Exposure to HIV and Post-Exposure Prophylaxis [5] and Harari regional state Dire Dawa administrative council [18]. The difference could be due to difference in study population this study only included health professionals with direct patient care. But those studies included non health professional auxiliary staffs.

And it is lower than a study from South Africa on blood borne pathogen exposure risk among surgeon (70\%) (9), and finding from similar research on high risk for occupational exposure to HIV and utilization of PEP in Pune India (81.5\%). This could be due to difference in study populations and methodology respectively. Those were only on physicians (surgeons) in the South African study and prospective study design in the Pune India study [15].

But this finding contradicts with the with finding in the Jimma zone Oromia region south west Ethiopia (60.3\%) [1]; the same study population, comparable sample size, lower service and lower mean age of the respondents but high needle and sharp injury as compared to this research. However the estimated rate of needle stick injury in this study was lower as compared to WHO estimation for developing countries (2.10 injuries per person per year). The most common reason for sustaining needle stick injuries in this study was due to patient sudden movement $(43.7 \%)$ followed by recapping $27 \%$. Despite the current national infection prevention recommendation, not to recap needle it is still a common practice.

This study also detected high level of exposure to blood and body fluids $(82.5 \%, 74.9 \%)$ respectively as compared to a research done Harari regional state and Dire Dawa administrative $(28.8 \%, 20.2 \%)$ respectively and a research finding in Jimma zone Oromia region south west Ethiopia $(44.3 \%, 39.1 \%)$ respectively this may be due to low service year of the respondents of these area as compared to study's

\begin{tabular}{|c|c|c|c|c|c|}
\hline \multirow[b]{2}{*}{ Variables } & \multirow{2}{*}{$x^{2}$} & \multirow{2}{*}{ P-value } & \multirow{2}{*}{ AOR } & \multicolumn{2}{|c|}{ 95.0\% Cl.For AOR } \\
\hline & & & & Lower & Upper \\
\hline Age Category & 1.793 & .616 & & & \\
\hline $20-29$ & .265 & .606 & .536 & .050 & 5.735 \\
\hline $30-39$ & .772 & .380 & .379 & .043 & 3.305 \\
\hline $40-49$ & .009 & .923 & 1.099 & .163 & 7.396 \\
\hline Profession & 2.300 & .681 & & & \\
\hline Physician & 1.802 & .179 & 4.960 & .479 & 51.400 \\
\hline Health officer & .358 & .549 & 2.258 & .157 & 32.479 \\
\hline Nurse & .726 & .394 & 2.589 & .290 & 23.072 \\
\hline Midwife & .363 & .547 & 2.114 & .185 & 24.189 \\
\hline Risk of HIV infection & 1.186 & .553 & & & \\
\hline Yes & .224 & .636 & 1.710 & .185 & 15.789 \\
\hline No & .133 & .716 & .573 & .029 & 11.414 \\
\hline Training pep (yes) & 5.124 & .024 & 2.864 & 1.152 & 7.122 \\
\hline Score knowledge & .718 & .698 & & & \\
\hline Good knowledge & .638 & .425 & .257 & .009 & 7.220 \\
\hline Fair knowledge & .475 & .491 & .311 & .011 & 8.620 \\
\hline Service year category $(<35)$ & .219 & .639 & 1.480 & .287 & 7.644 \\
\hline Constant & .532 & .466 & .171 & & \\
\hline
\end{tabular}

Table 7: Multivariate logistic regression analysis of PEP utilization among health professionals in Mekelle town. 
participant service year and multiple procedures that can predipose health professionals to many body fluids and blood could not undertake in these area as many of the study participants were from health center.

The use of PEP among the exposed health professionals in this study is $19.6 \%$ it is in line with previous studies done in Ethiopia [1] and to a research done in the UK on PEP for HIV knowledge and experience of junior doctors [14]. But it is lower than a research finding on high risk for occupational exposure to HIV and utilization of PEP in Pune, India (72\%) among the exposed [15] and HIV prone occupational exposures epidemiology and factors associated with initiation of postexposure prophylaxis in London (79.8\%) among the exposed [16-25]. The difference may be probably different in type of exposure in these the type exposures were needle stick injuries while in this study the type exposures were needle stick, exposure to blood and body fluids. And in this study health professionals started PEP after test result of the source patient. But in those studies after significant exposure they started PEP and they wait for test results of the source patient.

For those who started PEP all of them had HIV counseling and testing before commencing PEP. This is true with the Ethiopian national guideline on management of occupational exposure against HIV.

According to national infection prevention, WHO and CDC recommendations PEP treatment as much as possible should be initiated immediately after exposure within 1-2hours. Despite of these recommendations the mean time to initiate PEP drugs in this study was 13.35 hours.

$80.6 \%$ of those started PEP completed their drugs. $19.4 \%$ of those who started discontinue the drugs due to adverse effects of the drugs. But this is lower than a drug discontinuation due to adverse effects of the drugs found in a teaching hospital, Pune India [15] and research report from PEP for HIV knowledge and experience of junior doctors in the UK. But this finding is in line with the estimation of CDC (1747\%) proportion of health professionals taking PEP after occupational exposure to HIV positive sources didn't complete a full 4 week course of therapy because of an inability to tolerate the drugs [6].

Concerning the post PEP HIV test result $93.3 \%$ were negative only one (3.448\%) was positive. As already known PEP is not $100 \%$ effective. The protective capacity of PEP drugs when taken properly is $80 \%$. So this finding is consistent with of estimation of protection capacity of PEP [12].

Results of both Bivariate and multivariate analysis confirmed that PEP utilization was significantly associated with training of health professionals on PEP (AOR=2. 864, 95\% CI 1.152-7.122). But no other research findings that show the impact of training on PEP utilization?

\section{Conclusions}

From the findings of the study the following conclusions were summarized.

- Occupational exposures were common among health professionals in the study area;

Occupational exposures to blood, body fluids, to needle stick and sharp injuries and mucocutaneous were $82.5 \%, 74.9 \%, 49.1 \%$ and $42.7 \%$ respectively.

The recapping of the needle was still a common practice; $27 \%$ of the needle stick injuries were due to recapping
- Some unnecessary measures were taken by health professionals after needle stick or sharp injuries; $11.9 \%$ of the injuries squeezed the site for more bleeding

- The utilization of PEP among the exposed health professionals was low.

- According to this finding certain number of health professionals could not get PEP drugs for exposures that occur during duty time

- The mean time to initiate the first PEP drug after exposure was 13.35hours

- $25 \%$ of the exposed respondents were negligent to seek PEP the study

\section{Recommendations}

Based on the findings summarized under the conclusion, the following recommendations were forwarded.

1. Health facilities may strengthen and integrate infection with routine services through providing training to all health professionals so as to decrease the high occupational exposures.

2. Health facilities are expected to have the standard protocol for infection prevention to avoid unnecessary practices such as recapping of needle and squeezing for more bleeding.

3. To increase the utilization of PEP, all health professionals are better to be trained on PEP

4. Health facilities are expected to be the center of available PEP drugs all the time.

5. To be on the safe side after exposure, PEP is better to be started immediately.

6. The government could introduce Health insurance for health professionals

7. Further study with designs out of cross-sectional study design is recommended.

\section{References}

1. Tebeje B, Hailu C (2010) Assessment of HIV Post-Exposure Prophylaxis Use Among Health Workers of Governmental Health Institutions in Jimma Zone, Oromiya Region, Southwest Ethiopia. Ethiop J Health Sci 20: 55-64.

2. Management of healthcare workers exposed to HIV.

3. CDC (1998) Public Health Service Guidelines for the Management of HealthCare Worker Exposures to HIV and recommendations for Post exposure Prophylaxis. MMWR 47: 1-28.

4. Julian G, Maggy T (2005) occupational exposure for HIV. The Albion Street, center Sydney Australia, WHO/ILO.

5. Tadesse A (2008) Assessment of Health Care Workers Occupational Exposure to HIV and Post-Exposure Prophylaxis in Health Centers and Hospitals of Addis Ababa, Addis Ababa -Ethiopia. Addis Ababa university, Ethopia.

6. Adelisa L, Denise M, Lisa A, Walid H, Clara S (2005) Updated U.S public health service guide lines and recommendation for post exposure prophylaxis. MMWR 54: 1-24.

7. Landovitz RJ, Currier JS (2009) Clinical practice. Postexposure prophylaxis for HIV infection. N Engl J Med 361: 1768-1775.

8. (2010) Ministry of health the HIV prevalence and current status of the epidemic in Ethiopia.

9. Elayne K, Sats P, Paul G, Janine J (2011) Blood born pathogen, exposure risk among South African surgeons. The South African gastroenterology review 9. 
Citation: Gebreslase T, Buruh G (2014) HIV Post-Exposure Prophylaxis Use and Associated Factors among Health Professionals of Governmental Health Institutions in Mekelle Town, Tigray Ethiopia, Cross-Sectional Study. J AIDS Clin Res 5: 313. doi:10.4172/2155-6113.1000313

10. Antony S, Fauci H, Clifford L (2008) Human immune deficiency virus disease, AIDS and related disorders. Anthony Fauci, Eugene Braunwald, Dennis Kasper, Stephen Hauser, Dan Longo, et al, Harrison's principle of internal medicine 17edition. New York, 1137-1203.

11. Ashebir Ayalew (2010) Assessment of knowledge attitude and practice and factors associated with PEP service utilization among health professionals, Addis Ababa, EPHA.

12. Ministry of Health (2008) Guideline for management of opportunistic infections and Anti retro viral therapy in adolescent and adults in Ethiopia, federal ministry of health Ethiopia.

13. Annil M, Vishal R, Harsh S, Kulbir S (2007) Post exposure Prophylaxis after Occupational HIV Exposure.

14. Chen MY, Fox EF, Rogers CA (2001) Post-exposure prophylaxis for human immunodeficiency virus: knowledge and experience of junior doctors. Sex Transm Infect 77: 444-445.

15. Gupta A, Anand S, Sastry J, Krisagar A, Basavaraj A, et al. (2008) High risk for occupational exposure to HIV and utilization of post-exposure prophylaxis in a teaching hospital in Pune, India. BMC Infect Dis 8: 142.

16. Makary MA, Al-Attar A, Holzmueller CG, Sexton JB, Syin D, et al. (2007) Needlestick injuries among surgeons in training. N Engl J Med 356: 2693-2699.

17. van der Maaten GC, Nyirenda M, Beadsworth MJ, Chitani A, Allain T, et al. () Post exposure prophylaxis of HIV transmission after occupational injuries in Queen Elizabeth Central Hospital, Blantyre, Malawi, 2003 - 2008. Malawi Med J 22: 15-19.
18. Shiferaw T (2012) Occupational Exposure of Health Care Workers to Bloodborne and Body Fluid Infectious Agents in Harari Regional State and Dire Dawa Administrative Council, Ethiopia.

19. Saraswati V, Rashmi J, Prabha A, Animesh J (2011) Awareness of Post Exposure Prophylaxis Guidelines against Occupational Exposure to HIV Among Post Graduate Residents at Mangalore, India. Int J Med Public health 1: $51-56$.

20. Alenyo R, Fualal J, Jombwe J (2009) Knowledge, Attitude and Practices of Staffs towards Post-exposure Prophylaxis for HIV Infection at Mulago Hospital in Uganda. East Cent Afr j Surg 14: 99-102.

21. WHO (2007) Post exposure prophylaxis to prevent HIV Infection. Joint WHO/ ILO guidelines on post-exposure prophylaxis (PEP) to prevent HIV infection.

22. Elizabeth H, Philippa E (2007) Occupational exposure to HIV and the use of post-exposure prophylaxis united kingdom.

23. Kennedy I, Williams S (2000) Occupational exposure to HIV and post-exposure prophylaxis in healthcare workers. Occup Med (Lond) 50: 387-391.

24. Julia L (2003) Occupational exposure to HIV in health care setting. N Engl J Med 27: 348-349.

25. Zenner D, Tomkins S, Charlett A, Wellings K, Ncube F (2009) HIV prone occupational exposures: epidemiology and factors associated with initiation of post-exposure prophylaxis. J Epidemiol Community Health 63: 373-378. 